\title{
Anthelmintic Resistance and Its Mechanism: A Review
}

\author{
Workye Fissiha' \\ Mebrie Zemene Kinde ${ }^{2}$ \\ 'Department of Epidemiology and Public \\ Health, College of Veterinary Medicine \\ and Animal Sciences, University of \\ Gondar, Gondar, Amhara Regional State, \\ Ethiopia; ${ }^{2}$ Department of Veterinary \\ Biomedical Sciences, College of \\ Veterinary Medicine and Animal Sciences, \\ University of Gondar, Gondar, Amhara \\ Regional State, Ethiopia
}

\begin{abstract}
Helminths are a various types of parasites causing a major health problem for animals in different parts of the globe. Control of helminthiasis has largely relied on the use of pharmaceutical anthelmintics. Unfortunately, the exhaustive use of anthelmintic drugs has led to a serious and dramatic level of anthelmintic resistance. Anthelmintic resistance is a heritable loss of sensitivity of an anthelmintic in a parasite population that was in the past susceptible to the same anthelmintic. The development of anthelmintic resistance is evident to different helminths of almost every animal species and to different groups of anthelmintic in several continents. Frequent treatment, underdosing, genetics of the parasite, and targeting and timing of mass treatment are predisposing factors for anthelmintic resistance. Upregulation of cellular efflux mechanisms, an increase in drug metabolism, a change in drug receptor sites that reduces drug binding or the functional consequences of drug binding, and a decrease in drug receptor abundance through reduced expression within the parasite are the main mechanisms of anthelmintic resistance. In vivo method like fecal egg count reduction test and in vitro method such as egg hatch assays, larval motility test, larval development test and PCR can be used for the detection of anthelmintic resistance. Proper utilization of anthelmintic drugs, using combined anthelmintic and applying other alternatives are essential strategies to slow down the development of anthelmintic resistance. As anthelmintic resistance is a serious challenge throughout the world, proper utilization of the existing anthelmintics and reducing dependence on anthelmintics should be implemented to reduce its challenge.
\end{abstract}

Keywords: anthelmintic, development, helminths, mechanism, resistance

\section{Introduction}

Helminths are a group of worms which cause a major health problem on animals worldwide. Even though controlling pastures for domestic animals could reduce the impact of parasites, these techniques are not adequate to get rid of these parasites. Control of helminthiasis has largely relied on the use of pharmaceutical anthelmintics which can represent the single largest part of the expenditure on animal health in many countries. ${ }^{1}$

Currently, anthelmintic drugs are the basis for the management of infection caused by veterinary helminths, and will probably remain so for the future due to the general lack of antiparasitic vaccines. In the last 50 years, the chemical control of parasites in animals was very successful due to the remarkable effectiveness with more than 95\% parasite reduction, the general good safety margin, the broad spectrum nature and the fair costs of the anthelmintics. Sadly, application of anthelmintic drugs in an intensive way has led to a serious and dramatic level of
Correspondence: Mebrie Zemene Kinde Department of Veterinary Biomedical Sciences, College of Veterinary Medicine and Animal Sciences, University of Gondar, Gondar, Amhara Regional State, Ethiopia

Tel +251918518866

Email zemenemebrie@gmail.com 
anthelmintic resistance (AR), principally in cattle, sheep, goat, and equine gastrointestinal nematodes. ${ }^{2}$ The increasing development of AR in parasites of livestock is threatening animal health and production worldwide. ${ }^{3}$ Currently, there are three classes of anthelmintics most commonly used in small ruminants: the benzimidazoles (BZs), macrocyclic lactones (MLs) and cholinergic agonists (especially levamisole; LEV). AR has been reported in all classes of anthelmintics. ${ }^{2}$ The time that the development of resistance against an anthelmintic drug from its introduction looks like less than 10 years. ${ }^{4}$

AR development is a highly multifaceted process which is affected via the host, the parasite, type of anthelmintic and its utilization, animal management and climatic characteristics. Thus, enhancing the challenges for developing the controlling and preventing measures that could vary based on the animal production systems. The involvement of several factors, the challenges to develop new anthelmintics and the difficulty to reverse the resistant strains to susceptible are very important contributors of AR development. ${ }^{5}$ Even though AR is increasing from time to time and becomes a major challenge due to intensive application of anthelmintics, establishing effective alternative strategies to control the helminth infection have not been established so far. As a result, regular detection of $\mathrm{AR}$, understanding the predisposing factors and mechanisms of anthelmintic resistance is very important to slow the spread of resistance parasites. Hence, the main objective of this review is to highlight $A R$ and its predisposing factors, development mechanisms, detection methods, and strategies to delay development of AR.

\section{Anthelmintic Resistance and Its Mechanism \\ Definition of Anthelmintic Resistance}

$\mathrm{AR}$ is a heritable loss of sensitivity of an anthelmintic in a parasite population that was in the past susceptible to the same anthelmintic. It is considered that $\mathrm{AR}$ is present when a higher proportion of the parasite individuals within a population are able to lose sensitivity to doses of an anthelmintic than in a normal population of the same species and it is transmitted from generation to generation. ${ }^{6}$

According to Nipane et al, ${ }^{7}$ there are three types of AR, namely cross resistance, side resistance and multiple resistance. Cross resistance is the first type of resistance in which a parasite strain is able to tolerate the therapeutic doses of anthelmintics which are unrelated chemically or anthelmintics having different mechanism of action. The second type of resistance is side resistance and it is a condition in which the resistance to an anthelmintic is due to selection by another anthelmintic having a similar mechanism of action. Resistance among benzimidazoles anthelmintics is considered as an example of side resistance. It is reported that strains which are resistant to levamisole also develop side resistance to morantel. Development of resistance to two or more anthelmintic having a similar or different mechanism of action because of either selection by each group independently or by side resistance is the third type of anthelmintic resistance and it is known as multiple resistance.

\section{Current Situations of Anthelmintic Resistance}

The development of AR is evident to different helminths of almost every animal species and to different groups of anthelmintic in several continents. ${ }^{8}$ In Europe, the results of many scientific studies indicate, to variable extents, an increase of helminth resistance against the wellknown groups of anthelmintic which are benzimidazoles, tetrahydropyrimidines, and imidazothiazoles and macrocyclic lactones. The cross-sectional study conducted by Mickiewicz et $\mathrm{al}^{9}$ revealed the presence of AR to BZ, ML, and LEV on Polish goat farms, in which the resistance is widespread to $\mathrm{BZ}$ and ML, while the resistance to LEV is at a low level. Potârniche et $\mathrm{al}^{2}$ also reported the resistance of gastrointestinal nematodes against MLs and BZs, in goats in Romania, which is the first report in the country. In addition, isolated reports revealed the occurrence of helminth resistance to the most recent classes of anthelmintics (for instance, Haemonchus contortus has developed resistance to monepantel, $\mathrm{C}$, and an aminoacetonitrile derivative). ${ }^{10}$ Investigations have shown that resistance has developed to anthelmintics in a short period of time after launch to the market and even in some countries, several sheep and goat farms have been closed due to anthelmintic resistance. ${ }^{11}$ The time that the development of resistance against an anthelmintic drug from its introduction looks like less than 10 years. In sheep, resistance development against imidazothiazoles, tetrahydropyrimidines and avermectin milbemycins classes occurred within three to nine years. The severity and range of this problem, particularly with respect to multidrug resistance in the nematode population, is expected to 
increase. ${ }^{4}$ Some authors reported the widespread incidence of multidrug-resistant populations of Haemonchus contortus, Teladorsagia and Trichostrongylus to benzimidazoles, imidazothiazoles and macrocyclic lactones in sheep throughout Europe. ${ }^{12}$

With extensive use of the anthelmintic in many tropical and parasite-endemic countries, the graveness of parasite resistance is exaggerated. The trend in the use of excessive medication in animals is also posing threats to public health. The governmental bodies are either lacking understanding or underestimating the regime of this emerging issue. ${ }^{13}$ Broad spectrum anthelmintics which are most commonly used for treatment of livestock GIT helminth infection in Ethiopia fall under the three anthelmintic families, which are benzimidazoles (eg, albendazole and triclabendazole), imidazothiazoles (like tetramisole and levamisole) and macrocyclic lactones (eg, ivermectin). Unregulated and inappropriate anthelmintic use contributed to the failure to abate livestock gastrointestinal parasitism, and instead resulted in appearance of AR in various nematode parasites under different geographical areas in Ethiopia. ${ }^{14}$ Wondimu and Bayu ${ }^{15}$ from Haramaya, Ethiopia, reported the presence of multidrug-resistant gastrointestinal nematodes in goats against albendazole, tetraclozan, ivermectin, and tetramisole, of which Trichostrongylus spp., Teladorsagia spp. and Haemonchus spp. were commonly identified genera on post-treatment culture. From Limpopo Province, South Africa, high prevalence of AR against gastrointestinal nematodes infecting sheep was reported by Mphahlele et al. ${ }^{16}$

\section{Factors Contributing for Development of Anthelmintic Resistance}

The efficiency of modern anthelmintics is about $99 \%$ against vulnerable strains. A small number of tolerant parasites are the most resistant component of the population. These survival parasites are disposed into the environment and contaminate the pasture that leads to development of a majority of resistant generations which result in development of AR due to selection pressure. The rate AR development is affected through several factors, from those frequency of treatments is the most important. ${ }^{17}$

\section{Frequency of Treatment}

This is an important determinant of the speed of development of AR. When an anthelmintic is given for treatment more frequently, the development of resistance to the anthelmintic is faster. The fundamental principle of selection for $\mathrm{AR}$ is that treatment offers the surviving parasites a reproductive and replication advantage over the susceptible parasites, for about two to three weeks after the anthelmintic is administered. ${ }^{18}$

\section{Targeting and Timing of Mass Treatment}

It has been indicated that providing mass prophylactic treatment contributed to the development of AR in helminths. However, it is possible to delay the development of resistance by treating about $80 \%$ of the flock. ${ }^{17}$

\section{Anthelmintic Dose Rates}

Administration of incorrect and inappropriate anthelmintic dose is one of the main several factors that may contribute to the development of AR. Visual weight estimation is the most commonly applied method to determine the dose rate of an anthelmintic in particular and a drug in general in veterinary medicine, which is often inappropriate and can lead to underdosing. In turn, this underdosing allows the survival of heterozygous resistant worms and therefore, contributes to selection of resistant strains. ${ }^{19}$

\section{Genetics}

Resistance parasites are pre-existing in the population of a parasite. AR is currently recognized as a pre-adaptive phenomenon in which the resistance alleles are present in the parasite population before it has ever been uncovered to the anthelmintic in query. Natural selection retains the resistance alleles at a low frequency in the absence of anthelmintics because the resistance alleles render the worms carrying them less suited for survival than completely susceptible worms. The introduction and continuing use of an anthelmintic, however, gives resistant worms a survival advantage. This allows them to reproduce at a faster rate than susceptible worms, resulting in an increase in the frequency of worms with a resistance phenotype within the population. Eventually, the frequency of worms with a resistance phenotype rises to the point where anthelmintic resistance is said to have appeared or developed. Only homozygous worms tolerate a proper dose of anthelmintic when anthelmintic resistance is a recessive trait in worms. The anthelmintic kills parasites that are heterozygous. ${ }^{1}$

\section{Mechanism of Anthelmintic Resistance}

Understanding the mechanisms of resistance can help researchers better predict how quickly resistance will 
emerge, as well as provide a tool for studying parasite biology and therapeutic targets. Anthelmintic resistance mechanisms typically include (1) upregulation of cellular efflux mechanisms, (2) an increase in drug metabolism, (3) a change in drug receptor sites that reduces drug binding or the functional consequences of drug binding, or (4) a decrease in drug receptor abundance through reduced expression or another route for downregulation. Between helminth species, the link between the aforementioned alterations and resistance differs. ${ }^{20}$

\section{Macrocyclic Lactone Resistance}

Resistance to individual medications in the class, such as ivermectin, is included in macrocyclic lactone resistance. The term avermectin and milbemycin resistance is frequently used to denote macrocyclic lactone resistant worm populations because of its long history of use. The most likely targets of ML treatment are ligand-gated chloride channels and it has been proposed that mutations in the genes that encode these proteins may confer AR. The occurrence of mutations in GluClRs was first reported in papers on the mechanism of ivermectin resistance in parasitic worms. In ivermectin and moxidectin-resistant Haemonchus contortus isolates, an allele of a GluClasubunit gene was found more often, implying that a mutation in this gene was linked to ML resistance. ${ }^{21,22}$

Protein transporters, especially P-glycoproteins (Pgps) serve as an efflux mechanism to transport molecules across the cell membrane thus lowering their intracellular concentration. This prevents the drug from reaching its target site. Ivermectin resistance associated with Pgps has been reported in H. contortus. PGP-2 is the most consistently identified Pgp to be associated with ML resistance. ${ }^{22}$ Enzymes which are involved in drug metabolism are another non-specific mechanism most probably leading to macrocyclic lactone resistance. Recently, Yilmaz et $\mathrm{al}^{23}$ reported increased expression of CYP34/35 in a multidrug-resistant $H$. contortus isolate compared to a susceptible isolate.

\section{Resistance to Benzimidazoles}

The mechanism of benzimidazole anthelmintic resistance has been definitively linked to alterations in B-tubulin. Resistance to BZ can be caused by the phenyalanine200tyrosine change in isotype I B-tubulin. ${ }^{24}$

Even a single amino acid mutation in the tubulin protein causes benzimidazole binding to be blocked in resistant nematodes. BZ resistant gastrointestinal nematode (GIN) species have been linked to three nonsynonymous single nucleotide polymorphisms (SNPs) in the isotype 1-tubulin gene. The most prevalent SNP causes a phenylalanine to tyrosine substitution at position 200 (F200Y), while the others cause a phenylalanine to tyrosine substitution at position 167 (F167Y) or a glutamic acid to alanine substitution at position $198(\mathrm{G} 198 \mathrm{Y})(\mathrm{E} 198 \mathrm{~A}){ }^{25}$

\section{Imidothiazoles and Tetrahydropyrimidines Resistance} The function of nicotinic acetylcholine receptors (nAChRs), particularly the L-type subset of these receptors, which is preferentially activated by levamisole and pyrantel, has been the focus of research into the causes of resistance to nicotinic agonist medicines. ${ }^{26}$ When these L-nAChRs are activated, they cause neuromuscular depolarization and spastic paralysis. In diverse species of Trichostrongylid nematodes, there is some indication that alterations to the target site within nematodes are the likely mechanism of resistance to levamisole and pyrantel. Reduced expression levels of genes coding for nACh subunits, which make up the receptor, have been linked to levamisole and pyrantel resistance of $H$. contortus and A. caninum, respectively. In isolates of $H$. contortus, T. colubriformis, and T. circumcincta, the presence of shortened forms of two receptor subunits (acr-8b as a truncated form of acr- $8 \mathrm{a}$, and unc- $63 \mathrm{~b}$ as a truncated form of unc-63a) has also been linked to resistance. ${ }^{27,28}$

\section{Methods for Detection of Anthelmintic Resistance}

Before settling on an AR diagnosis, a number of criteria must be considered. To begin, keep in mind that a range of illnesses might cause clinical indications that are similar to those linked with parasitism. Second, anthelmintic treatments may fail to control nematodes for reasons other than resistance. Failure in these circumstances is frequently attributable to issues such as malfunctioning drenching equipment or underdosing due to an incorrect body weight estimation. With the rise in $\mathrm{AR}$, there is a greater demand for dependable and standardized detection methods. In vivo and in vitro approaches are used to detect and monitor $\mathrm{AR}^{29}$

\section{In vivo Methods}

\section{Fecal Egg Count Reduction Test (FECRT)}

The anthelmintic efficacy of a chemical is determined by comparing worm egg counts from the animal before and after treatment. This test has been thoroughly 
standardized, allowing it to be widely used. Resistance is evident when two requirements are met, according to FECRT: the percent reduction in egg count is less than $95 \%$, and the lower limit of its $95 \%$ confidence range is equal to or less than $90 \%$. A post-treatment egg count for benzimidazoles should be performed 10-14 days after the anthelmintic has been delivered. Because anthelmintic treatment can temporarily stop eggs from being laid without killing adult nematodes, itis a good idea to use it. ${ }^{30}$ Egg production may be decreased if the time between treatments is less than 10 days, resulting in an overestimation of anthelmintic efficacy with the benzimidazoles anthelmintic. As a result, collecting feces samples 10-14 days following treatment is recommended. Fecal samples should be taken less than 7 days after treatment if levamisole resistance is suspected. As a result, depending on the anthelmintic group, the duration between treatment and the second egg count varies: 7-10 days for benzimidazoles; 3-7 days for tetrahydropyrimidines and imidazothiazoles; $14-17$ days for macrocyclic lactones. ${ }^{12}$

\section{In vitro Methods}

\section{Egg Hatch Assays (EHAs)}

Benzimidazoles anthelmintic prevent embryonation and hatching of the eggs of nematode parasites. This technique has been established to detect resistance against this group of anthelmintic. The test is not suitable for the use of tetrahydropyrimidines, imidazothiazoles and macrocyclic lactones as they are not ovicidal. In the test, fresh eggs are placed in each well of a 24 multiwell plate, and several concentrations $(0.5,1,2,3,5 \mathrm{ppm})$ of the benzimidazoles are added after $48 \mathrm{~h}$ of incubation at $27^{\circ} \mathrm{C}$, the remaining eggs and hatched larvae are counted and the $\mathrm{LD}_{50}$ values are calculated. It is a more feasible method for testing benzimidazoles resistance. ${ }^{31}$

\section{Larval Development Test (LDT)}

This test is based on the capacity of larvae to survive and develop in varied anthelmintic medication concentrations. The development of larvae (from eggs of a pooled fresh feces sample in a sub-group of the flock) under various doses of the anthelmintic is examined in larval development tests. Incubation can be done either on a liquid or a solid nutritional medium (agar). AR against the major anthelmintic families is detected using this approach. Variations in $\mathrm{LD}_{50}$ (larval 50\% death) have been reported in this test depending on the timing of infection, especially when macrocyclic lactones (ML) are utilized. Some veterinary offices and regional veterinary laboratories offer this test. ${ }^{32}$

\section{Larval Motility Test (LMT)}

Larvae three are incubated at $25^{\circ} \mathrm{C}$ for $24 \mathrm{~h}$ in various concentrations of drug while in the dark. Then they are exposed to light for $20 \mathrm{~min}$ to stimulate those not paralyzed. After that the number of nonmotile larvae as proportion of the total larvae present at each drug concentration are calculated. ${ }^{33}$

\section{Polymerase Chain Reaction (PCR)}

The genotyping of resistant ( $\mathrm{rr}$ ) or susceptible ( $\mathrm{rS}$ and SS) adult worms or larvae is possible with this technology, which is based on the use of PCR. Worms can be genotyped for the mutation on $\beta$-tubulin residue 200 (phenylalanine to tyrosine), which is implicated in BZ resistance, by employing four primers in the same reaction mixture. ${ }^{34}$

\section{Management Strategies to Delay the Development of Anthelmintic Resistance}

The application of anthelmintics for the management of helminths of livestock for the last five decades has resulted in resistance development to each of the major anthelmintic classes. The development of new anthelmintics to manage resistance is a slow and costly process. Hence, it is extremely crucial to use the existing anthelmintics in a way that minimizes the impact of AR. ${ }^{20}$

For the aim of preventing parasite infection and or maintaining low infection pressure various management strategies such as pasture management and refugia. These would reduce the necessity of anthelmintic usage that could contribute to delay development of AR. The important actions which are required to slow down the progression of AR are proper utilization of anthelmintic, reduce dependence on anthelmintic, avoid the introduction of resistance onto a farm by treating purchased stock on arrival followed by a quarantine period, and maintain anthelmintic susceptible population of worms and test for anthelmintic resistance regularly. ${ }^{32}$

\section{Correct Use of Anthelmintics}

It is considered that there is a risk of AR development when helminth parasite populations are exposed to an anthelmintic drug. Surprisingly, the risk of resistance development increases during underdosing as well as a very frequent applications of anthelmintics which are grouped to the same class. Rotation of anthelmintic classes 
has been suggested to slow down the development of resistance. Application of a reliable diagnosis to determine the type of the worm, use of anthelmintics that are effective based on the diagnosis, following the label directions for correct dosing and administration are very important strategies to delay anthelmintic resistance. ${ }^{35}$

\section{Refugia}

Prevention of resistance must focus on slowing the accumulation of resistance alleles, and strategies to slow the development of resistance must be implemented early in the resistance evolution process, before clinical evidence of reduced drug efficacy emerges. This is best performed by adhering to policies that assure a sufficient level of refugium; a word used to indicate the fraction of a parasite population that is not exposed to a specific treatment, hence avoiding selection for resistance. ${ }^{1}$ Anthelmintic resistance is inherited in a worm population, and once resistance has been established, there is no reversion or loss of resistance. By permitting the conservation of susceptible individuals to dilute the progeny of resistant parasites that survive treatment, refugia limit the development of resistance. The pace of evolution toward resistance slows as the size of the refugium grows larger. ${ }^{36}$

\section{Use of Combined Anthelmintics}

As a way of slowing down the development of AR, it has been recommended the use of combined anthelmintics having a related spectrum of activity but different mode of action. Due to the development of imidothiazole and tetrahydropyrimidine resistance, recently, the interest to use a combination of different anthelmintic classes has been increased to control in the presence of resistant nematodes, and slow the development of resistance numbers. $^{37}$

\section{Other Options}

Reducing the frequency of anthelmintic usage is very useful to decrease the rate of AR development. Applying a better grazing management is a possible and helpful method to reduce the frequency anthelmintic use. Lowering of the stocking rate and the grazing period on the pastures, and applying mixed grazing among different animal species are key factors for a better grazing practice. Applying biological control is also a remarkable technique to reduce the use of anthelmintics. The main principle in biological control is using natural enemies that can eat/kill the parasites to decrease the infection level on pastures. ${ }^{38}$
These treatments do not attempt to eliminate free-living larval stages, but rather to lower them to a point where they have minimal clinical or subclinical impact while promoting an acquired immune response. ${ }^{39}$ Selecting genetically less vulnerable animals is one method that has been tried to lessen the helminth burden in animals. ${ }^{40}$

The development of efficient vaccines against intestinal parasites will allow antiparasitic medications to be used less frequently. Despite significant attempts to develop vaccinations to protect grazing animals from helminth infections, only a vaccine for the Dictyocaulus viviparus is currently commercially available. ${ }^{41}$

\section{Conclusion and Recommendations}

The intensive anthelmintic use for the management of helminths in livestock has led to the development of AR, which is a highly multifaceted process and affected by the treated animal, the parasite, type of anthelmintic and its utilization. Misuses of anthelmintic such as underdosing, treatment of all animals at the same time on the same farm, continued administration of the same anthelmintic, substandard quality, and frequent use of anthelmintic are very important contributors to AR development. Upregulation of cellular efflux mechanisms, an increase in drug metabolism, a change in drug receptor sites that reduces drug binding or the functional consequences of drug binding, and a decrease in drug receptor abundance through reduced expression within the parasite are the main mechanisms of anthelmintic resistance. Nowadays, apart from application of anthelmintics there are no other effective options to control parasitic helminths. In addition, the development of new anthelmintics to manage AR is a slow, as well as expensive, process. Hence, it is crucial to use the existing anthelmintics in a way that minimizes the impact of AR such as proper and combined utilization of anthelmintics and reducing dependence on anthelmintics. Regular detection and monitoring of $\mathrm{AR}$ development is also crucial.

\section{Disclosure}

The authors report no conflicts of interest in this work.

\section{References}

1. Shalaby A. Anthelmintic resistance; how to overcome it? Iran J Parasitol. 2013;8(1):18-32.

2. Potârniche AV, Mickiewicz M, Olah D, et al. First report of anthelmintic resistance in gastrointestinal nematodes in goats in Romania. Animals. 2021;11:2761. doi:10.3390/ani1110276 
3. Peña-Espinoza M. Drug resistance in parasitic helminths of veterinary importance in Chile: status review and research needs. Austral J Vet Sci. 2018;50:65-76. doi:10.4067/S0719-81322018000200065

4. Kaplan RM. Drug resistance in nematodes of veterinary importance: a status report. Trends Parasitol. 2004;20(10):477-481. doi:10.1016/ j.pt.2004.08.001

5. Mederos AE, Carracelas B, Minho AP, Fernández S, Sánchez J. Prevalence and factors associated with anthelmintic resistance in gastrointestinal nematodes of cattle: a systematic review and meta-analysis. J Vet Med Health. 2018;2:2.

6. Abbott A, Taylor L, Stubbings A. Technical manual for veterinary surgeons and advisers 4th edition; 2012.

7. Nipane SF, Mishra B, Panchbuddhe AN. Anthelmintic resistanceclinician's present concern. Vet World. 2008;1(9):281.

8. Baiak BHB, Lehnen CR, Rocha RA. Anthelmintic resistance in cattle: a systematic review and meta-analysis. Livest Sci. 2018;217:127-135. doi:10.1016/j.livsci.2018.09.022

9. Mickiewicz M, Czopowicz M, Moroz A, et al. Prevalence of anthelmintic resistance of gastrointestinal nematodes in Polish goat herds assessed by the larval development test. BMC Vet Res. 2021;17 (19):1-12. doi:10.1186/s12917-020-02721-9

10. Vanden R, Moll L, Kappert C, Vellema P. Haemonchus contortus resistance to 544 in sheep. Vet Parasitol. 2015;209:278-280. doi:10.1016/j.vetpar.2015.02.026

11. Erez MS, Kozan E. Anthelmintic resistance in farm animals. Kocatepe Vet J. 2018;11(3):322-330.

12. Papadopoulos E, Gallidis E, Ptochos S. Anthelmintic resistance in sheep in Europe: a 509 selected review. Vet Parasitol. 2012;189 (1):85-88. doi:10.1016/j.vetpar.2012.03.036

13. Ali Q, Rashid I, Ashraf K, Shabbir Z, Chaudhry U. Rationale to understand anthelmintic resistance in parasitic nematodes. $J A d v$ Parasitol. 2019;6(2):16-20.

14. Urga B, Feyera T. Anthelmintic resistance of gastrointestinal parasites in small ruminants; 2015. Available from: https://www.omicson line.org/open-access/anthelmintic-resistance-of-gastrointestinalparasites-in-small-ruminant. Accessed May 26, 2017.

15. Wondimu A, Bayu Y. Anthelmintic drugs resistance of gastrointestinal nematodes of naturally infected goats in Haramaya, Ethiopia. 2019:1-17. doi:10.21203/rs.2.13752/v1

16. Mphahlele M, Tsotetsi-Khambule AM, Moerane R, Komape DM, Thekisoe OMM. Anthelmintic resistance and prevalence of gastrointestinal nematodes infecting sheep in Limpopo Province, South Africa. Vet World. 2021;14(2):302-313. doi:10.14202/vetworld.2021.302-313

17. Jabbar A, Iqbal Z, Kerboeuf D, Muhammad G, Khan N, Afaq M. Anthelmintic resistance: the state of play revisited. Life Sci. 2006;79 (26):2413-2431. doi:10.1016/j.1fs.2006.08.010

18. Vercruysse J, Albonico M, Behnke M, Kotze AC, Prichard RK. Is anthelmintic resistance a concern for the control of human soil-transmitted helminths? Int J Parasitol Drugs Drug Resist. 2011;1(1):14-27. doi:10.1016/j.ijpddr.2011.09.002

19. Nielsen K, Fritzen B, Duncan L, Guillot J, Esker M, Dorschies P. Practical aspects of equine parasite control. Equine Vet J. 2010;42 (5):460-468. doi:10.1111/j.2042-3306.2010.00065.x

20. Sarai RS, Kopp SR, Coleman GT, Kotze AC. Drug-efflux and target-site gene expression patterns in Haemonchus contortus larvae able to survive increasing concentrations of levamisole in vitro. Int J Parasitol Drugs Drug Resist. 2014;4:77-84. doi:10.1016/j. ijpddr.2014.02.001

21. Kotze. A, Hunt W, Skuce P, von Samson-himmelstjerna G, Martin RJ. Recent advances in candidate-gene and whole-genome approaches to the discovery of anthelmintic resistance markers and the description of drug/receptor interactions. Int J Parasitol Drugs Drug Resist. 2014;4(3)::164-18. doi:10.1016/j.ijpddr.2014.07.007
22. Jessica SK. Anthelmintic Resistance in Equine Parasites: Anthelmintic Resistance in Equine Parasites: Mechanisms and Treatment Approaches. [theses and dissertations] Veterinary Science, degree of Doctor of Philosophy in the College of Agriculture, Food and Environment, University of Kentucky University of Kentucky Uknowledge; 2019:288.

23. Yilmaz E, Ramünke S, Demeler J, Krücken J. Comparison of constitutive and thiabendazole-induced expression of five cytochrome P450 genes in fourth-stage larvae of Haemonchus contortus isolates with different drug susceptibility identifies one gene with high constitutive expression in a multi-resis. Int $J$ Parasitol Drug. 2017;7:362-369. doi:10.1016/j.ijpddr.2017.10.001

24. Shayan P, Eslami A, Borji H. Innovative restriction site created PCR-RFLP for detection of benzimidazoles resistance in Teladorsagia circumcincta. Parasitol Res. 2007;100(5):1063-1068. doi:10.1007/s00436-006-0357-y

25. Haudhry U, Redman E, Raman M, Gilleard J. Genetic evidence for the spread of benzimidazoles resistance mutation across southern India from a single origin in the parasitic nematode Haemonchus contortus. Int $J$ Parasitol. 2015;45::721-8. doi:10.1016/j. ijpara.2015.04.007

26. Martin A, Robertson S, Buxton R, Beech C, Charvet C. Levamisole receptors: a second awakening. Trends Parasitol. 2012;28:289-296.

27. Sarai S, Steven R, Kopp M, et al. In vitro levamisole selection pressure on larval stages of Haemonchus contortus over nine generations gives rise to drug resistance and target site gene expression changes specific to the early larval stages only. Vet Parasitol. 2015;211:45-53.

28. Wolstenholme J, Fairweather I, Prichard R, von Samsonhimmelstjerna G, Sangster NC. Drug resistance in veterinary helminths. Trends Parasitol. 2004;20(10):469-476. doi:10.1016/j. pt.2004.07.010

29. Ihler CF. Anthelmintic resistance. An overview of the situation in the Nordic countries. Acta Vet Scand. 2010;52(Suppl 1):S24. doi:10.1186/1751-0147-52-S1-S24

30. Álvarez-sánchez M, Perez-Garcia J, Cruz-Rojo MA, Rojo-Vázquez FA. Real time PCR for the diagnosis of benzimidazole resistance in trichostrongylids of sheep. Vet Parasitol. 2005;129(3-4):291-298. doi:10.1016/j.vetpar.2005.02.004

31. Zajac A, Conboy G. Veterinary Clinical Parasitology. 7th ed. UK: Black well; 2006:19-20.

32. Teagasc A; 2010 https://waww.tegasc.ie/media/website/publications/ 2010/AnthelminticResistance.pdf. Accessed May 4, 2017.

33. Kohler P. Invited review the biochemical bases of anthelmintic action and resistance. Int J Parasitol. 2001;31:336-345. doi:10.1016/S00207519(01)00131-X

34. Elard L, Cabaret J, Humbert JF. PCR diagnosis of benzimidazole-susceptibility or -resistance in natural populations of the small ruminant parasite, Teladorsagia circumcincta. Vet Parasitol. 1999;80(3):231-237. doi:10.1016/S0304-4017(98)00214-3

35. Sargison N. Pharmaceutical control of end parasitic helminth infestations in sheep. Vet Clin North Am Food Anim Pract. 2011;27 (1):139-156. doi:10.1016/j.cvfa.2010.10.014

36. Sangste $\mathrm{C}, \mathrm{Rj} \mathrm{D}$. Anthelmintic resistance. In: Lee DL, editor. The Biology of Nematodes. Harwood; 2002:531-567.

37. Bartrama D, Dave M, Leathwick M, et al. The role of combination anthelmintic formulations in the sustainable control of sheep nematodes. Vet Parasitol J. 2012;186:151-158. doi:10.1016/j. vetpar.2011.11.030

38. Larsen M. Biological control of nematodes in sheep. J Anim Sci. 2006;84:E133. doi:10.2527/2006.8413_supplE133x

39. Waller P, Schwan O, Ljungström B, Rydzik A, Yeates G. Evaluation of biological control of sheep parasites using Duddingtonia flagrans under commercial farming conditions on the island of Gotland, Sweden. Vet Parasitol. 2004;126:299-315. doi:10.1016/j.vetpar.2004.08.008 
40. Stear M, Doligalska M, Donskow-Schmelter K. Alternatives to anthelmintic for the control of nematodes in livestock. Parasitology. 2007;134(02):139. doi:10.1017/S0031182006001557
41. Smith WD, Zarlenga D. Developments and hurdles in generating vaccines for controlling helminth parasites of grazing ruminants. Vet Parasitol. 2006;139:347-459. doi:10.1016/j.vetpar.2006.04.024

\section{Publish your work in this journal}

Infection and Drug Resistance is an international, peer-reviewed openaccess journal that focuses on the optimal treatment of infection (bacterial, fungal and viral) and the development and institution of preventive strategies to minimize the development and spread of resistance. The journal is specifically concerned with the epidemiology of

Submit your manuscript here: https://www.dovepress.com/infection-and-drug-resistance-journal antibiotic resistance and the mechanisms of resistance development and diffusion in both hospitals and the community. The manuscript management system is completely online and includes a very quick and fair peerreview system, which is all easy to use. Visit http://www.dovepress.com/ testimonials.php to read real quotes from published authors. 